\title{
Formation of biofilms of pathogenic bacteria and the effect of a new disinfectant
}

\author{
I.B. Pavlova ${ }^{1}$, A.B. Kononenko ${ }^{1, *}$, G.S. Tolmacheva ${ }^{1}$, G.G. Kardash ${ }^{2}$, and A.Yu. Rytsarev ${ }^{2}$
}

${ }^{1}$ All-Russian Research Institute of Veterinary Sanitation, Hygiene and Ecology - a branch of the Federal Scientific Center - All-Russian Research Institute of Experimental Veterinary Medicine named after K.I. Scriabin and Ya.R. Kovalenko, 123022 Moscow, Russia ${ }^{2}$ LLC "Sun Systems", 119421 Moscow, Russia

\begin{abstract}
The article presents results of experimental study of biofilm formation by pathogenic bacteria Pseudomonas aeruginosa, Salmonella enteritidis and Staphylococcus aureus. Using the method of investigation of microbiological objects by light and scanning electron microscopes, preserving their architectonics, process of biofilm formation and its development phases were shown. Morphological and biological features of the effects of a nanotechnology preparations containing Chlorhexidine-base were studied. Its bactericidal effect on biofilms of pathogenic bacteria was shown.
\end{abstract}

\section{Introduction}

According to modern concepts of biology of microorganisms, it is obvious that almost all bacteria, including those in human and animal bodies, form biofilms [1]. Biofilm is a community consisting of one or several types of bacteria, as well as fungi adhered to the surface and enclosed in the exopolymer matrix $[2,3]$. This form of existing protects bacteria from adverse effects of antibacterial factors, in particular, of disinfectants, and the immune system.

There are reports showing that bacteria isolated from biofilms have higher level of pathogenicity (virulence) compared to filmless species. The ability of bacteria to form biofilms on the surface of various medical instruments and equipment, catheters, etc. creates big medical problems $[4,5]$.

Ability of bacteria in biofilms to survive in presence of antibiotics in much higher concentration then standard therapeutic ones is well known. Possibility of a longterm survival of microorganisms in biofilms is due to heteromorphism and formation of $\mathrm{L}$-forms in uncultured state [6-9].

Problem of destroying or suppressing formation of biofilms is extremely important, since a wide range of antibacterial agents (antibiotics, disinfectants) do not always ensure the desired effect, and super-resistant forms of infectious pathogens can develop. Studies on the role of biofilms expand knowledge of their structural and functional features and allow to approach to many practical goals of destruction of infectious agent in organism and on environmental objects. It is necessary to develop methods to prevent bacteria from forming biofilms.

This article is devoted to study of the bactericidal activity of two samples of a new disinfectant - preparation containing Chlorhexidine-base provided by San Systems LLC (preparation 1 and preparation 2).

\section{Methods and materials}

San Systems LLC developed a new approach to creation of antimicrobial agents that can destroy biofilm matrix and kill microorganisms inside it.

Chlorhexidine is used in the form of salts - biacetate, bigluconate or dihydrochloride. It is known that Chlorhexidine acts on cell membrane, increasing its permeability. Acting as a cationic antiseptic having a high degree of affinity for cell walls it is rapidly adsorbed on the microbial wall. The idea of developing of a new drug presents new mechanism of the "attack" of molecules of a chemical biocide on microbial cell.

It is generally accepted to consider impact of individual molecules of a chemical substance on a microbial cell, but in reality biocidal solutions are existing in form of complex molecular associations which interact with bacterial cell structure and biofilm matrix [10].

Nanotechnology approach has been developed to create a "liquid crystalline phase" which is a matrix for encapsulating oligomeric molecules with specific functional chemical groups. In fact, destruction of biofilms and bacteria occurs at a controlled level of supramolecular structures. The technology allows us to encapsulate biocidal substances or drugs, while maintaining their native capacity. Preparation samples tested in this work contain well known in medical practice Chlorhexidine as an active substance in the form of its base. Chlorhexidine-base is insoluble. Using new patented technology, San Systems LLC managed to "solubilize", i.e.to place it inside amine oxide capsules that are soluble in water and other solvents.

Corresponding author: sanmicrobio@mail.ru 
Following microorganisms were used in the experiment: Pseudomonas aeruginosa, Salmonella enteritidis, and Staphylococcus aureus.

A new method has been developed for study of microorganisms at the population level without violating architectonics with parallel use of light and electron scanning microscopes. This approach allows us to study and visualize various manifestations of heteromorphism, L-transformation and formation of stable and unstable Lforms.

In view to study phases of biofilm formation and their structure, we used method of growing microorganisms on coverslips in liquid and semi-liquid nutrient media. We used 24-48-hour microorganisms cultures in the S-form, grown in solid or liquid nutrient media. Suspension of bacteria at a concentration of $10^{5}$ $\mathrm{CFU} / \mathrm{ml}$ (according to the turbidity standard) in the amount of $5 \mathrm{ml}$ was shaken using a Vortex apparatus and introduced into Petri dishes with $20 \mathrm{ml}$ of meat-peptone broth. Sterile fat-free coverslips were placed on sterile slides and immersed in a liquid nutrient medium in Petri dishes. The material was incubated in a thermostat for 24-48-72 hours at $37{ }^{\circ} \mathrm{C}$. The coverslips were removed with tweezers and placed in Petri dishes with paper filters on the bottom (the preparations were not dried). In order to preserve natural architectonics, it was fixed in vivo by vapor of $25 \%$ of glutaraldehyde for 2-3 hours. Preparations were colored by vapor of 2-4\% aqueous solution of $\mathrm{OsO} 4$ for 2-3 minutes. For fixing by vapors, a solution in form of drops was applied on the surface of a paper filter placed on top of a Petri dish. After treatment with osmic acid vapors, the biofilms with bacteria turned yellow or brown. Preparations were examined using a scanning electron microscope after dehydration with propylene oxide vapor and gold ion sputtering (Methodological recommendations for studying biofilms of conditionally pathogenic and pathogenic microorganisms in vitro, All-Russian Research Institute of Veterinary Sanitation, Hygiene and Ecology, 2017). This method differs from usually used methods for these purposes as mobility of the mucous or gel components of the biofilms is not disturbed. During fixation and washing of research objects in a liquid solution, structure of biofilms undergoes irreversible changes.

Effect of disinfectants (Chlorhexidine-base No. 1 and 2 preparations) was studied on biofilms grown within 48 and 72 hours. Solutions at a concentration of 1: 100 was exposed on biofilms for 30 and 60 minutes. The control was unaffected biofilms. To study survival of bacteria membrane filters (pore diameters of $0.22 \mu \mathrm{m}$ and 0.45 $\mu \mathrm{m})$ placed in Petri dishes in solid nutrient media were used. To identify L-transformation processes, a filter with a pore diameter of $0.22 \mu \mathrm{m}$ (sandwich) was placed under the filter with a pore diameter of $0.45 \mu \mathrm{m}$. A "sandwich" of two membrane filters was placed on the surface of a solid nutrient medium. Bacterial culture was applied in the form of a drop in the center of the filter. Cultivation was carried out for $24-48$ hours at $37^{\circ} \mathrm{C}$. Then, the number of colonies on the upper filter was visually evaluated, the lower filter with a pore diameter of $0.22 \mu \mathrm{m}$ was placed on the surface of a fresh, dense nutrient medium and cultivated under the above conditions for 5-7 days. Colonies grown on the surface of membrane filters were fixed by vapors of $25 \%$ glutaraldehyde and dehydrated twice with propylene oxide vapors than located on copper plates and sprayed with gold using a Hitachi-E-102 device (Japan). Bacterial cells remain on the upper filter, and small, unstable or stable L-forms formed during the Ltransformation process move to the lower filter.

In the experiment was fixed absence of visual growth on membrane filters. Bacterial morphology was examined using an AxioImager A1 optical microscope (Carl Zeiss, Germany) and a TM 4000 scanning electron microscope (SEM) (Hitachi, Japan).

\section{Results}

Study of the morphology of biofilm formation showed that gram-negative bacteria (Pseudomonas aeruginosa) adhere to the coverslip with subsequent formation of filamentary structures and formation of various sizes and shapes of clusters.

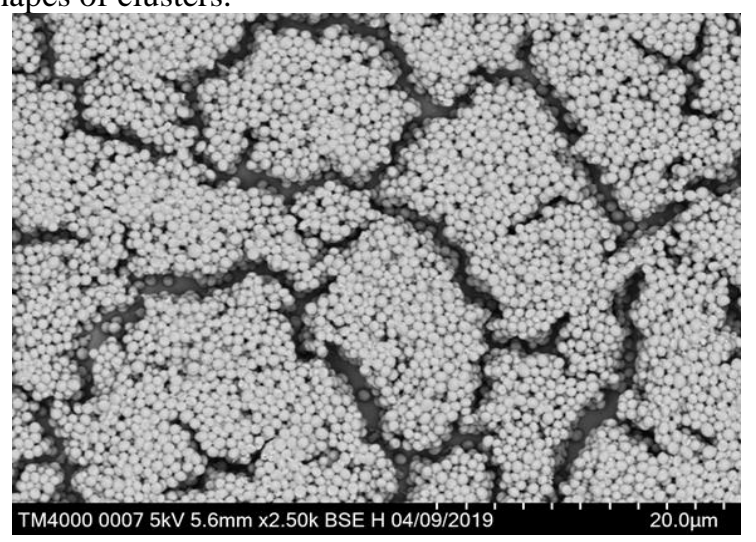

Fig. 1. Formation of S. aureus clusters. Inside the clusters, there is a biofilm with bacteria. SEM $\times 2500$

The example of S. aureus shows that during cultivating of populations of pathogenic bacteria in a liquid medium, it can be seen that on the surfaces of thin coverslips, a monolayer culture represented by single bacteria in the $\mathrm{S}$-form is formed in the beginning, than clusters and biofilms are formed (Fig. 1).

Figure 2 shows that 48 hours after the formation, P. aeruginosa growth was accompanied by formation of a dense biofilm. In some areas, single bacteria developing on the surface are visible, which indicates possibility of formation of the next layer of biofilm and therefore a multilayer. In this case, multilayer will be determined by type of bacteria, composition of nutrient medium, time of development of the biofilm and impact of adverse factors. Presence of pathogenic strains of P. aeruginosa in alginate causes high resistance to antibacterial and disinfectant drugs. With a large increase in SEM, heteromorphic cells of a spheroplastic, protoplast type of various sizes with various manifestations of Ltransformation are revealed in fragments of the population under the film and between the clusters (Fig. 3). 


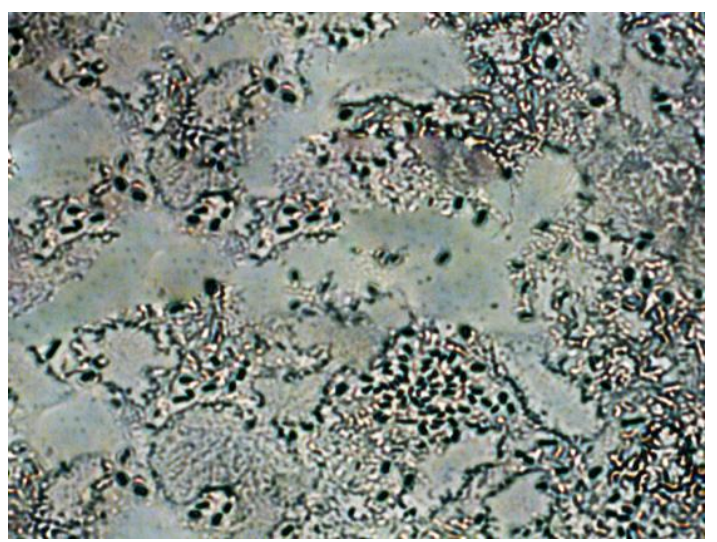

Fig. 2. Formation of a P. aeruginosa biofilm; on the surface, there are single bacteria emerging from the film. Optical microscopy, 10, 40

When examined $\mathrm{S}$. enteritidis with a scanning microscope, both single adhered bacteria and populations grouped in clusters are visible. Often channels that are involved in the metabolism and regulation of oxygen supply are identified inside forming biofilms.

Thus, using three different microorganisms, we have shown general rules of biofilm formation. Survival strategy of various pathogenic bacteria consists in their evolution by creation of biofilms in which bacteria are at early stages in vegetative form ( $\mathrm{S}$-form) with future transition to heteromorphism with various manifestations of L-transformation [6].

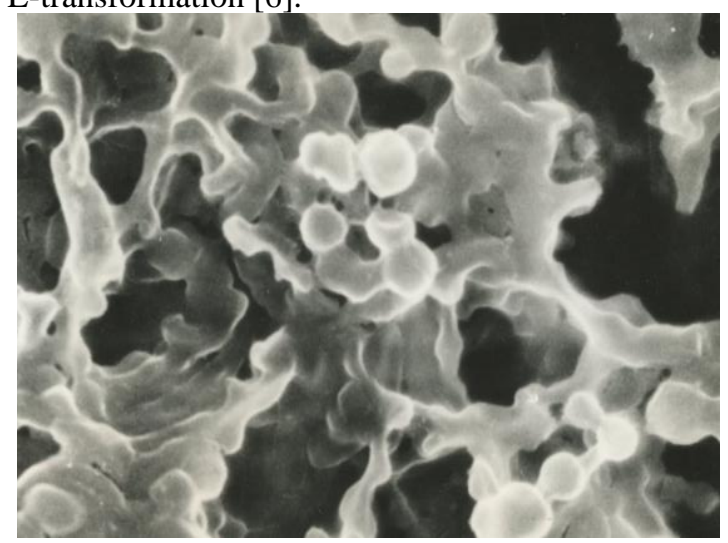

Fig. 3. Fragment of formation of S. enteritidis clusters of spheroplast and protoplast cells included in the biofilm. SEM, $\times 5000$

L-transformation process is accompanied by formation of small cells with a size of $0.2-0.3$ microns stable and unstable L-forms. The latter make up 20$25 \%$ of the total population. Under favorable conditions, they are reversed to their original state with preservation of pathogenicity and other biological features.

Biofilm structure can have different composition, depending on the type of pathogenic bacteria having a certain structure of the cell wall and containing peptides, lipids and other components.

Under certain conditions, a multilayer biofilm may develop. This process is associated with emergence of single bacteria on the surface of the upper layer. Then, filamentous structures are formed by bacteria, creating clusters with an exopolysaccharide matrix in which bacteria multiply and this contributes to the formation of subsequent biofilm layer (Fig. 4).

Study of biofilms of gram-positive and gramnegative bacteria indicates a sequence of phases of development and existence of bacterial populations.

We investigated Chlorhexidine base containing preparations. The experiment was accompanied by the control (Fig. 5).

The effect of the preparations (preparation 1 and preparation 2) on bacterial population at a concentration of $1: 100$ and an exposure time of 30 minutes was destruction of biofilm structure which lost its integrity and was detected as separate fragments. At the same time, one can see accumulation of altered cells devoid of cell wall, as evidenced by formation of round cells of sphero- and protoplast types capable of forming stable and unstable L-forms (Fig. 6).

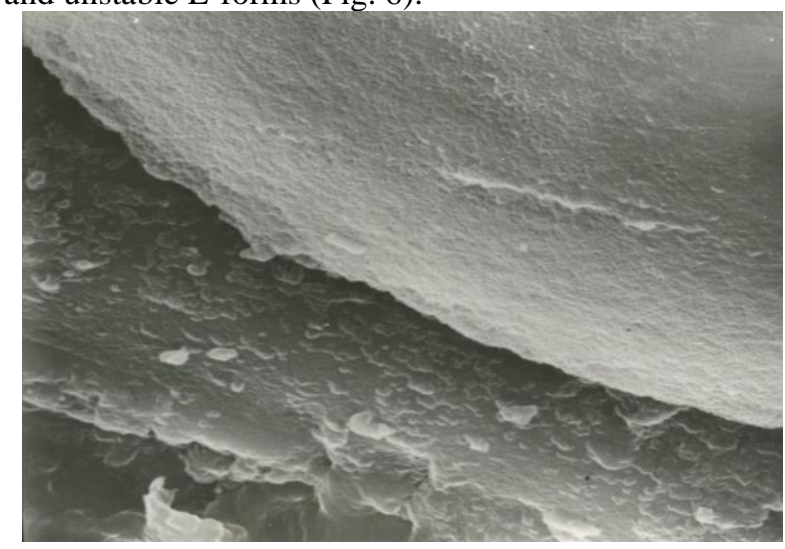

Fig. 4. Multi-layer biofilm S. enteritidis 72 hours after cultivation. Single cells are visible on the upper layer.

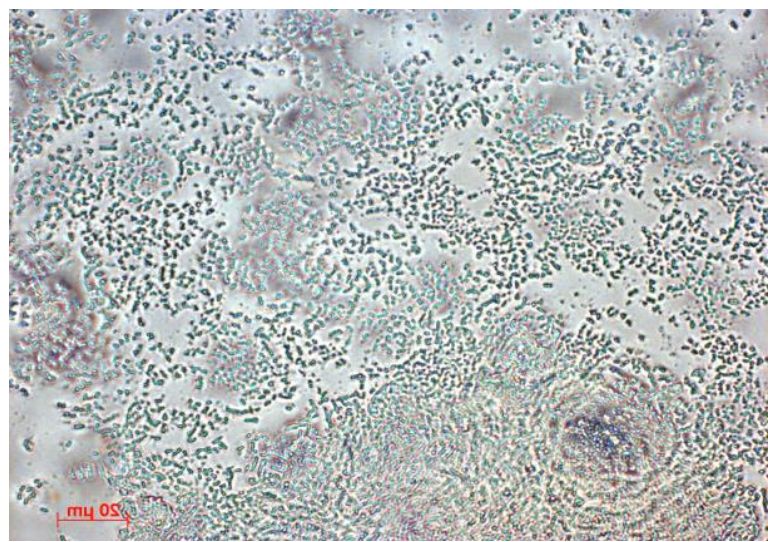

Fig. 5. The monolayer culture S. enteritidis with forming biofilms in clusters. Control (48 hours). Optical microscopy, approx. 10, vol. 40

Obtained data confirm action mechanism of Chlorhexidine base on cell wall and cytoplasmic membrane. At the same time, some of cells die and part of the population goes into a state of heteromorphism with formation of cells of spheroplast and protoplast type and L-forms. Bactericidal effect depends on time of exposure to the preparation. $60 \mathrm{~min}$ after, complete destruction of biofilm was revealed, while the bulk of cells died, which represent bactericidal effect. Impact of Chlorhexidine base destroyed cell wall and cytoplasmic membrane (Fig. 7). Significant destruction of biofilms 
and bacterial populations under influence of the preparations indicates bactericidal effect which was confirmed by the experiment on the survival of pathogenic bacteria exposed to the preparations in the same concentration.

The experiment showed typical growth of bacterial cultures on membrane filters in the control, while after 30 and 60 minutes of exposure, no visual growth was detected on the filters (Fig. 8).

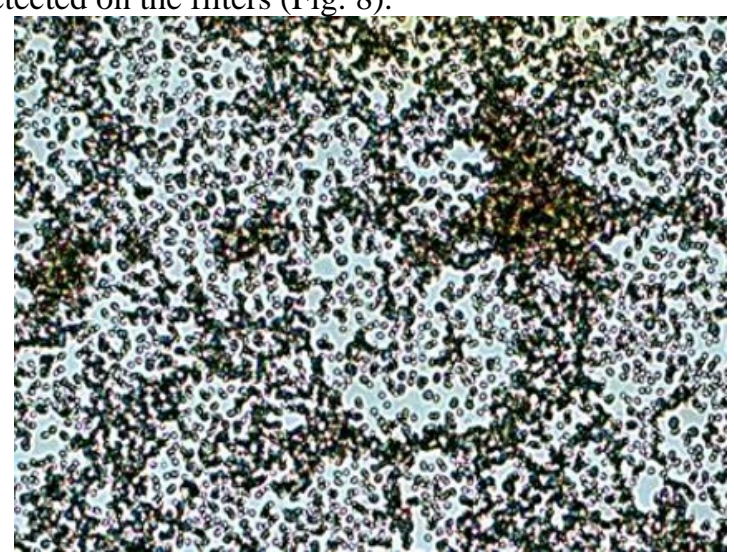

Fig. 6. Effect of the preparation 1 (concentration 1: 100) acting on S. enteritidis biofilms for 30 minutes. Remains of a biofilm with many round cells - spheroplasts are visible. Optical microscopy, approx. 10, vol. 40

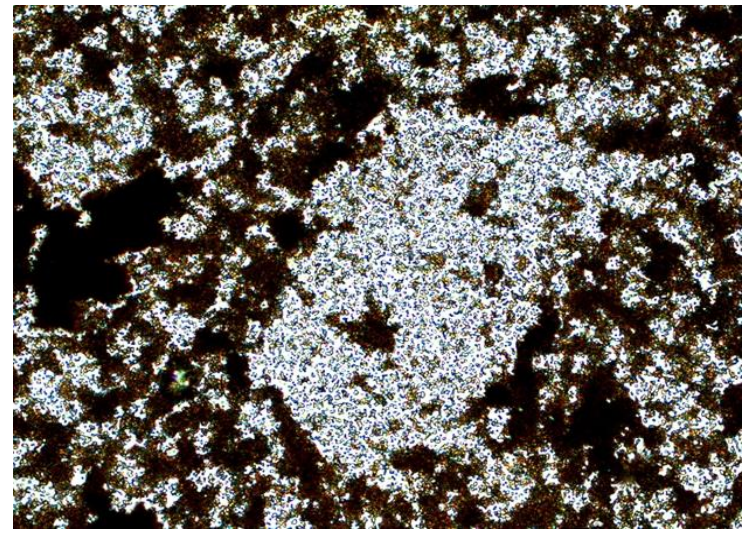

Fig. 7. Effect of the preparation 1 (concentration 1: 100) on S. aureus biofilms during 60 minutes. Destruction of biofilms, clusters, complete disintegration of bacterial cells inside the biofilm can be observed. Optical microscopy, approx. 10, vol. 40

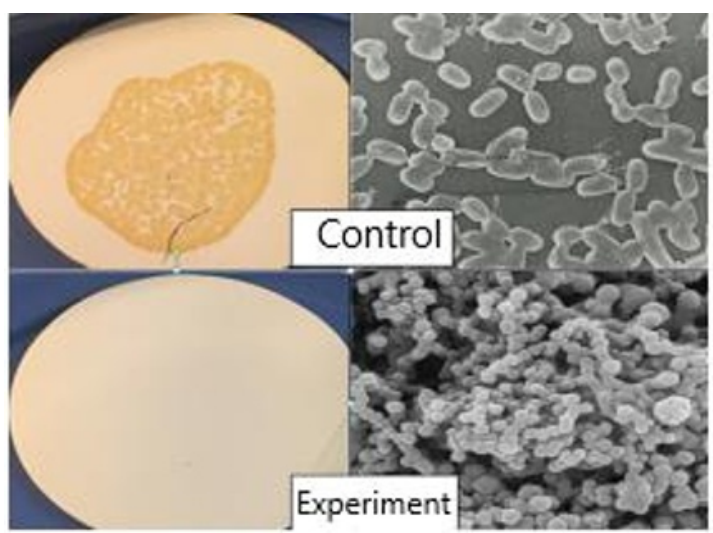

Fig. 8. "Sandwich" of two membrane filters. Control: abundant growth of S.enteritidis in the center of the filter is visible.
Experiment: visual absence of visible growth. In the control, population of adhered bacteria is visible; in the experiment, heteromorphism of spherical-type cells and small L-forms were revealed.

During long-term cultivation of the lower filters, after their transfer to fresh nutrient media to study possible reversion of unstable L-forms, there was no increase in the microbial population which proves bactericidal effect of the preparations.

\section{Conclusion}

Thus, we can conclude that the preparations have bactericidal effect on microbial populations inside biofilms. It is important to note destructive effect on biofilms in which bacteria enjoys increased resistance.

It gives us reason to believe that the tested form of Chlorhexidine base obtained by nanotechnology method can serve as a base for creation of new effective disinfectants in view to solve the problem of sanitary welfare in veterinary and medical practices. Our method of sampling for electron and light microscopy in the process of biofilm formation allowed us to study morphology of bacterial populations in this process without violating their architectonics.

This work allowed us to show the rules of the process of biofilm formation on a model of pathogenic bacteria populations with and without exposure to Chlorhexidine base.

\section{References}

1. O.V. Rybalchenko, V.M. Bondarenko, O.G. Orlova, Ultrastructure of microbial biofilms in intercellular relationships of bacteria in communities, J. of Microbiol., Epidemiol. and Immunol., 4, 87-92 (2014)

2. S.V. Maltsev, G.Sh. Mansurova, What is biofilm? Pract. Med., 5(53), 7-10 (2011)

3. T.A. Smirnova Structural and functional characteristics of bacterial biofilms, Microbiol., 79(4), 453-446 (2010)

4. L.V. Lagoon, Yu.V. Atanasova, D.V. Tapalsky, The formation of biofilms in pathogens of acute and chronic pyelonephritis, ZhMEI, 3, 18-23 (2013)

5. A.I. Nozhevnikova, E.A. Bochkova, V.K. Plaunov, Multi-species biofilms in ecology, medicine and biotechnology, Microbiol., 84(6), 623-644 (2015)

6. I.B. Pavlova, A.B. Kononenko, D.A. Bannikova, G.S. Tolmacheva, E.M. Lenchenko, Patterns of development of biofilms of bacteria at different phases of their formation in vitro, Probl. of Veter. Sanitat., Hygiene and Ecol., 4(28), 56-62 (2018)

7. I.V. Chebotar, A.N. Mayansky, E.D. Konchakova, A.V. Lazareva, V.P. Chistyakova, Antibiotic resistance of biofilm bacteria, Clin. Microbiol. and Antimicr. Therapy, 14(1), 51-58 (2012)

8. A.N. Mayansky, I.V. Chebotar, Staphylococcal biofilms: structure, regulation, rejection, J. of 
Microbiol., Epidemiol. and Immunobiol., 1, 101-108 (2011)

9. V.K. Plakunov, E.A. Strelkova, M.V. Zhurina, Persistence and adaptive mutagenesis in biofilms, Microbiol., 79(4), 447-458 (2010)
10. L.V. Didenko, G.G. Kardash, T.A. Smirnova et al., Study of the mechanism of action of tertiary alkylamines on clinical isolates of Escherichia coli and Staphylococcus aureus, Disinfect. Busin., 91(1), 32-36 (2015) 